\title{
The Feasibility of Scoring Systems for the Prediction of Mortality in Patient with Fournier's Gangrene: A retrospective analysis of 53 Patients
}

Serkan TAYAR ( $\sim$ tayarserkan61@hotmail.com )

Erzurum Regional Training and Research Hospital

Murat KARTAL

Erzurum Regional Training and Research Hospital

Yaşar ÇÖPELCi

Erzurum Regional Training and Research Hospital

Ayetullah TEMiz

Erzurum Regional Training and Research Hospital

Research Article

Keywords:

Posted Date: October 25th, 2021

DOl: https://doi.org/10.21203/rs.3.rs-964078/v1

License: (1) This work is licensed under a Creative Commons Attribution 4.0 International License. Read Full License 


\section{Abstract \\ Purpose}

Fournier's gangrene $(\mathrm{FG})$ is a polymicrobial disease that affects the skin and subcutaneous tissue and is life-threatening. This study aimed to evaluate the feasibility of scoring systems in predicting mortality in FG patients.

\section{Material and Method:}

53 patients diagnosed with FG and operated between March 2008-August 2020 in our clinic were included. These patients' demographic findings, laboratory values, Fournier's Gangrene Severity Index (FGSI), Uludağ Fournier's Gangrene Severity Indexi (UFGSI), the Sequential Organ Failure Assessment (SOFA), and Age-Adjusted Charlson Comorbidity Index (ACCI) scoring system values were recorded and analyzed regarding how these scoring systems affected mortality.

\section{Results}

$11(21 \%)$ of the 53 patients died, and $42(79 \%)$ survived. The mean age of the patients was $53.64 \pm 17.2$ years. The mean age of the surviving patients was $49.64 \pm 15.26$ years, while the mean age of the patients who died was $68.91 \pm 15.96(p=0.002)$. Of the laboratory parameters, only creatinine elevation was significant in demonstrating mortality $(\mathrm{p}=0.001)$. When the impact of scoring systems on mortality was evaluated, it was determined that the increase in $\mathrm{FGSI}(P=0.000), \operatorname{UFGSI}(P=0.000), \operatorname{SOFA}(P=0.000)$, and $\mathrm{ACCl}(\mathrm{P}=0.003)$ values were directly related to mortality and statistically significant.

\section{Conclusion}

The FGSI, UFGSI, SOFA, and ACCI scoring systems used in our study were correlated with mortality for FG. $\mathrm{ACCl}$ had the highest diagnostic confirmation value. This was followed by SOFA, FGSI, and UFGSI, respectively. We recommend that these scoring systems be used to predict mortality for FG.

\section{Introduction}

Fournier's gangrene (FG) is a type of necrotizing fasciitis accompanied by thrombosis of the feeding arteries, significantly affecting the perianal and genitourinary regions. It is a life-threatening polymicrobial disease that causes gangrene in the skin and subcutaneous tissues, leading to symptoms of multiple organ failure and severe intoxication [1]. Perianal disease, urethral strictures, local anorectal, urogenital or perineal trauma, malignancies, and comorbid systemic diseases, especially Diabetes Mellitus, are often responsible for the etiology of Fournier's gangrene [2]. Although FG is rare, it is a disease that needs attention due to the development of life-threatening complications in a short time and the difficulty of 
early diagnosis [3]. Two main scoring systems are used to determine the severity of FG in clinical practice. Fournier's Gangrene Severity Index (FGSI), the first scoring system based on acute physiological and chronic health status, developed in 1995, is widely used [4]. Considering vital signs and laboratory parameters, FGSI helps predict the prognosis and mortality of the disease [4]. Another widely used scoring system is Uludağ Fournier Gangrene Severity Index (UFGSI), developed by Yılmazlar et al. in 2010. It predicts mortality by considering the patient's acute physiological condition, age, and disease prevalence [5].

In addition to the scoring systems mentioned above, the Sequential Organ Failure Assessment (SOFA) score [6] and Age-Adjusted Charlson Comorbidity Index (ACCI) [7] also predict the survival and prognosis of $\mathrm{FG}$ patients. There are limited publications in the literature in predicting the prognosis related to the SOFA score[6]. Age-Adjusted Charlson Comorbidity Index (ACCI), defined by Charlson et al., is a combination of age equivalence index and Charlson Comorbidity Index (CCI) (7).

A high ACCI score has been reported to be directly associated with poor outcomes and mortality in FG patients[2]. However, their reliability in predicting the severity of the disease and predicting mortality in FG patients has not yet been clarified.

This retrospective study aimed to evaluate the feasibility of FGSI, UFGSI, SOFA, ACCI, which are used to predict mortality by using demographic, clinical, and laboratory information of patients with FG, and we discussed the results in the light of current literature.

\section{Materials And Methods}

The single-center cohort study was designed as a retrospective study, and ethics committee approval was obtained from Erzurum Regional Training and Research Hospital (2021/ 02-40). There were 53 patients diagnosed with FG between March 2008 and August 2020 in Erzurum Regional Training and Research Hospital General Surgery Department. Patients who underwent surgical debridement and/or orchiectomy and/or colostomy were included in the study. All procedures were performed following the Declaration of Helsinki on human studies. Informed consent was obtained from all patients before the operation, and all data were scanned retrospectively from the hospital information system.

All patients diagnosed with localized necrotizing fasciitis infection in the perineal region and treated surgically were included in the study. Medical histories, symptoms, and physical examination findings of the patients were collected from the recorded data from the system. Patients were divided into two groups according to disease-related mortality within the first 30 days after diagnosis, and mortality (number of patients who died) was accepted as the primary outcome. Perianal abscesses without soft tissue infection and patients whose data were not available were excluded.

Intravenous fluid therapy was initiated for all patients after hospitalization, and broad-spectrum antibiotic therapy was administered according to the recommendations of the infectious diseases department. Appropriate antibiotic therapy was revised according to the culture results. After hemodynamic 
stabilization, all patients underwent surgical treatment, and tissue culture was obtained by debriding necrotic tissues. In patients with scrotum involvement, orchiectomy was also performed when deemed necessary. Colostomy was performed in eligible patient groups to prevent fecal contamination. All surgical procedures were performed by general surgery clinicians. The decision for orchiectomy and colostomy was taken by the primary surgeon.

\section{Variables}

Demographic information (age, gender), vital signs, body temperature (CO), heart rate (/min), respiratory rate $(/ \mathrm{min})$, mean arterial pressure $(\mathrm{mmHg})$, clinical and laboratory findings, hospital stay were recorded by looking at the medical records of the patients. FGSI, UFGSI, SOFA, and ACCI scores were calculated for each patient and recorded in the database.

To calculate the FGSI, body temperature, heart rate, respiratory rate, sodium, potassium, creatinine, white blood cell count, hematocrit, creatinine, and bicarbonate levels were measured and graded in the range of 0 to 4 . While calculating the UFGSI, the patient's age and disease extent were added in addition to the FGSI parameters. ACCI was calculated in the range of 1 to 6 by evaluating the presence of 19 medical conditions. The SOFA score, on the other hand, was graded from 1 to 4 according to the defect status by evaluating six organ systems (hematological, renal, circulatory, respiratory, hepatic, and central nervous system).

\section{Statistical Analysis}

Mortality was defined as death within the first 30 days after hospital admission, and the development of mortality was considered the primary outcome. The Shapiro-Wilk test was used in the distribution of continuous variables. Continuous variables were expressed as mean and standard deviation or median (1st-3rd interquartile ranges (IQR)) values. Student's t-test and Mann-Whitney test compared distributionbased continuous variables. The Chi-square test was used to compare categorical variables. ROC analysis curve was performed to determine cut-off values for different scoring systems used to predict mortality. Each optimal cut-off value was selected considering the highest sensitivity and high specificity, positive and negative predictive values.

\section{Results}

Eleven (21\%) of the 53 patients died, and 42 (79\%) survived. The mean age of the patients was $53.64 \pm 17.2$ years. The mean age of the surviving patients was $49.64 \pm 15.26$ years, while the mean age of the patients who died was $68.91 \pm 15.96$, and a statistically significant difference was found between them $(p=0.002)$. Demographic and clinical data are presented in Table I.

The patients were consulted in the presence of a fever of $38^{\circ} \mathrm{C}$ and above, scrotal and/or perineal malodorous discharge, swelling, edema, crepitation, and fluctuation. $62 \%$ of the patients had at least one 
additional comorbid disease, and the most common comorbidity was DM, which was observed in 13 (24.53\%) patients.

Laboratory parameters during hospitalization are summarized in Table I, and sodium, potassium, calcium, total protein, albumin, C-reactive protein, hemoglobin, and creatinine values were available. There was a significant difference in creatinine levels between deceased and surviving patients $(p=0.001)$.

FGSI, UFGSI, SOFA, ACCI scoring systems are shown in Table II. When the impact of scoring systems on mortality was evaluated, it was determined that FGSI( $P=0.000)$, UFGSI $(P=0.000)$, SOFA $(P=0.000)$, $\operatorname{ACCl}(P=0.003)$ elevations were directly related to mortality and statistically significant.

ROC analysis used in mortality-based sensitivity and specificity revealed that the optimum cut-off values for FGSI, UFGSI, SOFA, and ACCI were 3, 4, 4, 8, respectively. Cut-off values based on sensitivity and specificity are presented in Table III. In addition to the known efficacy of FGSI and UFGSI scoring systems in predicting mortality, SOFA and ACCI scoring systems have also been shown to be effective in predicting mortality. The area under the curve (AUC) for each scoring system is shown schematically in Figure I.

\section{Discussion}

Fournier's gangrene is a rare infection of the perianal and genital area. Despite advances in its etiology, diagnosis, and intensive care techniques, the mortality rate in Fournier's gangrene remains high. Due to the high mortality rates, the scoring systems used to predict mortality in these patients are of great importance. It has been demonstrated in previous studies that conditions such as diabetes mellitus, coagulopathy, severe sepsis, advanced age, prolonged hospital stay, malignancy, multiple organ failure, and alcoholism increase mortality in FG patients [8-10]. Despite advances in follow-up and medical treatment in modern intensive care units, mortality rates have been reported in the range of $30-50 \%$ [2]. The mortality rate in our study was $20.7 \%$.

Existing studies have indicated that advanced age increases mortality [11-13]. The most important reason regarding this condition seems to be related to the increasing number of predisposing diseases and the incidence of FG with aging. However, it was found that advanced age is not a direct factor affecting survival [14]. The mean age of the survivors in our study was $49.64 \pm 15.26$ years, while the mean age of those who died was $68.91 \pm 15.96$ years, and advanced age was found to impact mortality.

Diabetes mellitus is an important risk factor for necrotizing infections involving the lower extremities, perineum, head, and neck region $[15,16]$. The patients included in our study had cardiovascular disease, DM, hematological diseases, and malignancies. DM, which is frequently involved in the etiology of FG, was determined in approximately $25 \%$ of the patients included in the study. The rate of diagnosis of DM in patients who died was $53.85 \%$, and it was not statistically significant. In our study, high creatinine, one of the daily routine blood biochemistry tests, was another factor that increased mortality and was 
statistically significant. However, serum sodium, potassium, calcium, and hematocrit values were not statistically significant. Likewise, in the study of Tenorio $(\mathrm{N})$ and Mangwiron [11], it was revealed that high serum creatinine is a risk factor for mortality.

FGSI is a scoring developed by Laor et al. [4] using vital signs and some laboratory data to determine the severity and prognosis of FG in patients. FGSI is a modification of the APACHE II score. FGSI scores of 9 and above were found to be associated with mortality. However, Yılmazlar et al. [5] reported that this scoring system had some drawbacks in estimating prognosis and mortality, and the UFGSI scoring system was developed by adding age and extent of disease spread to this system. Both of these systems are frequently used to predict the prognosis and mortality of FG. In a study, both systems gave $100 \%$ sensitivity in sensitivity and specificity analysis. Specificity was 78\% for FGSI and 73\% for UFGSI [17]. While Czymek et al. reported $87 \%$ sensitivity and $77 \%$ specificity for FGSI in their study [18], in the study of Roghmann et al., these figures were reported as $85 \%$ and $67 \%$ for UFGSI [19]. In another study conducted by Yılmazlar et al. [20] in a series of 120 cases, no survivors were reported in patients with a UFGSI score of $\geq 9$. Threshold values for FGSI and UFGSI were 7 and 9 , respectively. In this study, the FGSI score was 2 in the survivors and 5 in the deceased. While the UGFSI score was 5 in the survivors, it was 10 in the deceased. These scores were considered to be directly related to mortality and statistically significant. Besides, in the ROC analysis used for mortality-based sensitivity and specificity, the cut-off values for FGSI and UFGSI were 3 and 4, respectively, the sensitivity was $81 \%$ and $54 \%$, and the specificity was $90 \%$ and $90 \%$, respectively. The AUC value was 0.859 for FGSI and 0.785 for UGFSI. We found that both scoring systems are practical markers in predicting mortality in parallel with the literature.

This study also used SOFA and ACCI scoring systems, which we assumed to affect mortality. The SOFA score is a scoring system used to calculate organ systems' defect status by grading from 1 to 4 . Few studies have been performed in the literature on predicting prognosis and mortality in this scoring system FG. In a recent study in our country, the SOFA score was 1 in the survivors and 4 in the deceased, and it was found to be directly related to mortality and statistically significant. Also, in the ROC analysis used, the cut-off value was 4 , while the sensitivity and specificity rates were $90 \%$ and $88 \%$, respectively [21]. In another study, it was reported that low mean SOFA scores were significantly associated with the probability of primary wound closure. In the study, the SOFA score was also 1.8 for those who survived and 3.8 for those who died [6]. In this study, we found the SOFA score to be 1 in the survivors and 3 in the deceased, and we found that it was directly related to mortality and was statistically significant. Also, in the ROC analysis used, the cut-off value was 4 , while the sensitivity and specificity rates were $81 \%$ and $88 \%$, respectively.

On the other hand, the ACCl score is a scoring system calculated in the range from 1 to 6 , evaluating the presence of 19 medical conditions into account. In two studies, the ACCI score was 3 and 3 , respectively, in the survivors, and 5 and 6 , in the deceased. In both studies, the ACCI score was found to be statistically significant in predicting mortality [22-23]. In a recent study of Usta et al. [21] with 60 patients, the ACCI score was found to be 3 in the survivors and 5 in the deceased, and the difference was found to be statistically significant. In the same study, the cut-off value was 5 in the ROC analysis used for ACCI, 
while the sensitivity and specificity rates were $30 \%$ and $98 \%$, respectively [21]. Our study determined the $\mathrm{ACCl}$ score to be 2 in the survivors and 4 in the deceased. The difference was statistically significant. In the ROC analysis used, the cut-off value was 8 , while the sensitivity and specificity rates were $100 \%$ and $88 \%$, respectively.

The retrospective nature of our study and the relatively low number of patients were the major limitations of our study. However, the inclusion of SOFA and ACCI as a scoring system for FG was the biggest challenge.

In conclusion, FG is a type of necrotizing fasciitis that affects the perianal and genitourinary regions, accompanied by thrombosis of the feeding arteries. Mortality rates still range between $30-50 \%$. There is still no standard parameter or scoring system to predict prognosis and mortality in the follow-up. In this study, we revealed that advanced age and high creatinine are significant parameters in predicting mortality. Also, we found that the FGSI, UFGSI, SOFA, and ACCI scoring systems used in our study were associated with mortality for FG. ACCI had the highest diagnostic confirmation value. This was followed by SOFA, FGSI, and UFGSI, respectively. We recommend that these scoring systems be used to predict mortality for FG.

\section{References}

1. Aliev, S. et al. Fournier disease in surgeon's practice.Khirurgiia, 2008(11): p.58-63.

2. Doluoğlu, Ã. G. et al. Overview of different scoring systems in Fournier's Gangrene and assessment of prognostic factors. Turkish Journal of urology, 42 (3), 190 (2016).

3. Chernyadyev, S. A. et al. Fournier's gangrene: literature review and clinical cases. Urologia internationalis, 101, 91-97 (2018).

4. Laor, E. et al. Outcome prediction in patients with Fournier's gangrene. The Journal of urology, 154 (1), 89-92 (1995).

5. Yilmazlar, T. et al. Fournier's gangrene: an analysis of 80 patients and a novel scoring system. Techniques in coloproctology, 14 (3), 217-223 (2010).

6. Lauerman, M. et al. Definitive Wound Closure Techniques in Fournier's Gangrene. Am. Surg, 84 (1), 86-92 (2018).

7. Charlson, M. E. et al. A new method of classifying prognostic comorbidity in longitudinal studies: development and validation. Journal of chronic diseases, 40 (5), 373-383 (1987).

8. Abbott, S. et al. Predictors of outcome in palliative colonic stent placement for malignant obstruction. Journal of British Surgery, 101 (2), 121-126 (2014).

9. Morpurgo, E. \& Galandiuk, S. Fournier's gangrene. Surgical Clinics, 82 (6), 1213-1224 (2002).

10. Paty, R. \& Smith, A. D. Gangrene and Fournier's gangrene. Urologic Clinics of North America, 19 (1), 149-162 (1992). 
11. Mangwiro, T. I. \& Lazarus, J. Fournier's gangrene: outcome analysis and prognostic factors. S Afr J Surg, 56 (3), 43-46 PMID: 30264942. (2018 Sep).

12. Arora, A., Rege, S., Surpam, S., Gothwal, K. \& Narwade, A. Predicting Mortality in Fournier Gangrene and Validating the Fournier Gangrene Severity Index: Our Experience with 50 Patients in a Tertiary Care Center in India. Urol Int, 102, 311-318 https://doi.org/10.1159/000495144 (2019).

13. Erdoğan, A. et al. Simple scoring system for prediction of mortality in Fournier's gangrene. Eur J Trauma Emerg Surg. 2016 Aug;42(4):513-518. doi: 10.1007/s00068-015-0572-2. Epub 2015 Sep 11. Erratum in: Eur J Trauma Emerg Surg. 2017 Sep 11;: PMID: 26362534.

14. Martinez-Rodriguez, R., Ponce de Leon, J., Caparros, J. \& Villavicencio, H. Fournier's gangrene: a monographic urology center experience with twenty patients. Urol Int, 83 (3), 323-328 (2009).

15. Brook, I. \& Frazier, E. H. Clinical and microbiological features of necrotizing fasciitis. Journal of Clinical Microbiology, 33 (9), 2382-2387 (1995).

16. Wong, C. H. et al. Necrotizing fasciitis: clinical presentation, microbiology, and determinants of mortality. JBJS, 85 (8), 1454-1460 (2003).

17. Üreyen, O., Acar, A., Gökçelli, U., Atahan, M. K. \& IIlhan, E. The usefulness of FGSI and UFGSI scoring systems for predicting mortality in patients with Fournier's gangrene: a multicenter study. Turkish Journal of Trauma and Emergency Surgery, 23 (5), 389-394 (2017).

18. Czymek, R. et al. Fournier's gangrene: is the female gender a risk factor? Langenbecks Arch Surg, 395, 173-180 (2010).

19. Roghmann, F. et al. Isthere a need for the Fournier 's gangrene severity index? Comparison of scoring systems for outcome prediction in patients with Fournier's gangrene. BJU International, 110, 359365 (2012).

20. Yılmazlar, T. et al. Fournier's gangrene: Review of 120 patients and predictors of mortality. Ulus Travma Acil Cerrahi Derg, 20, 333-337 (2014).

21. Usta, M. A. et al. (2020). Scoring Systems for the Prediction of Mortality in Patient with Fournier's Gangrene: an Analysis of 60 Patients. Indian Journal of Surgery,1-7.

22. Girgin, R., Cinar, O., Bulut, E., Akduman, B. \& Mungan, N. A. The role of the Platelet Mass Index (PMI) as a new prognostic factor in Fournier's gangrene. African Journal Of Urology, 24 (3), 226-232 (2018).

23. Marín, A. G. et al. Predictive factors for mortality in Fournier's gangrene: A series of 59 cases. Cirugía Española (English Edition), 93 (1), 12-17 (2015).

\section{Tables}

Table I. The comparison of demographic features, laboratory findings, and clinical outcomes of patients with Fournier's gangrene according to the mortality 


$\begin{array}{llll}\text { Total } & \text { Survivor } & \text { Non-Survivor } & \begin{array}{l}\text { P- } \\ \text { value }\end{array} \\ (n=53) & (n=42) & (n=11) & \end{array}$

\begin{tabular}{|c|c|c|c|c|c|}
\hline Age (year) ${ }^{a}$ & $\begin{array}{l}\text { Mean } \pm \\
\text { SD }\end{array}$ & $53.64 \pm 17.2$ & $49.64 \pm 15.26$ & $68.91 \pm 15.96$ & 0.002 \\
\hline \multirow[t]{2}{*}{ Sex (male) $)^{b}$} & Female & 15 (28.30) & 14(93.33) & $1(6.67)$ & 0.149 \\
\hline & Male & $38(71.70)$ & $28(73.68)$ & $10(26.32)$ & \\
\hline
\end{tabular}

\section{Comorbidities $^{b}$}

Cardiovascular

8 (15.09)

3(37.5)

$5(62.5)$

NA

Diabetes mellitus

13(24.53)

6(46.15)

7(53.85)

NA

Hematological

9(16.98)

2(22.22)

7(87.78)

NA

Malignity

3(5.66)

1(33.33)

2(66.67)

NA

\section{Laboratory Findings}

Sodium (mmol/L) ${ }^{\text {a }}$

$\begin{array}{llll}137.75 \pm 6.84 & 137.4 \pm 6.2 & 139.09 \pm 9.13 & 0.367\end{array}$

Potassium (mmol/dL) ${ }^{a}$

$4.22 \pm 0.70$

$4.22 \pm 0.69$

$4.25 \pm 0.77$

0.938

Calcium (mg/dL) ${ }^{\text {a }}$

$8.95 \pm 0.85$

$8.96 \pm 0.88$

$8.93 \pm 0.8$

0.930

Total protein $(\mathrm{g} / \mathrm{dL})^{a}$

$7.16 \pm 0.90$

$7.16 \pm 0.92$

$7.19 \pm 0.9$

0.852

Albumin $(\mathrm{g} / \mathrm{dL})^{\mathrm{a}}$

$3.86 \pm 0.82$

$3.95 \pm 0.76$

$3.56 \pm 1$

0.380

C-reactive protein

$(\mathrm{mg} / \mathrm{dL})^{a}$

$23.42 \pm 21.42$

$23.41 \pm 20.05$

$23.47 \pm 27.21$

0.503

Hemoglobin $(\mathrm{g} / \mathrm{dL})^{\mathrm{a}}$

$14.23 \pm 3.04$

$14.75 \pm 2.38$

$13.46 \pm 2.92$

0.134

Creatinine $(\mathrm{mg} / \mathrm{dL})^{\text {a }}$

$0.96 \pm 0.31$

$0.88 \pm 0.25$

$1.3 \pm 0.35$

0.001

Orchiectomy ${ }^{b}$

7(13.21)

5(71.42)

2(28.57)

NA

Colostomy ${ }^{b}$

6(11.32)

4(66.66)

2(33.33)

NA

Length of hospitalization ${ }^{c}$

8.68(2.019.0)

8(3-19)

9(2-17)

0.700

NA: not applicate, a: Mean and standard deviation, b: n (\%), c: Median (IQR) 
Table II. The comparison of scoring systems for patients with Fournier's gangrene according to the mortality

\begin{tabular}{lllll} 
& $\begin{array}{l}\text { Total } \\
(\mathbf{n}=\mathbf{5 3})\end{array}$ & $\begin{array}{l}\text { Survivor } \\
(\mathbf{n}=\mathbf{4 2})\end{array}$ & $\begin{array}{l}\text { Non-Survivor } \\
(\mathbf{n}=\mathbf{1 1})\end{array}$ & P value \\
\hline FGSI & $3.0[1.0-7.0]$ & $2.0[1.0-5.0]$ & $5.0[3.0-7.0]$ & $\mathbf{0 . 0 0 0}$ \\
\hline UFGSI & $6.0[3.0-15.0]$ & $5.0[3.0-10.0]$ & $10.0[8.0-15.0]$ & $\mathbf{0 . 0 0 0}$ \\
\hline SOFA & $2.0[1.0-4.0]$ & $1.0[1.0-4.0]$ & $3.0[1.0-4.0]$ & $\mathbf{0 . 0 0 0}$ \\
\hline ACCI & $2.0[1.0-6.0]$ & $2.0[1.0-5.0]$ & $4.0[1.0-6.0]$ & $\mathbf{0 . 0 0 3}$
\end{tabular}

All data are presented in Median values [Min-Max]

FGSI: Fournier Gangrene Severity Index, UFGSI: Uludag Fournier Gangrene Severity Index, SOFA:

Sequential Organ Failure System, ACCl: Age-Adjusted Charlson Comorbidity Index

Table III. The receiver operating characteristics (ROC) analysis of scoring systems for predicting the mortality of patients with Fournier's gangrene

\begin{tabular}{|c|c|c|c|c|c|c|c|c|}
\hline & $\begin{array}{l}\text { Cut- } \\
\text { off }\end{array}$ & $\begin{array}{l}\text { Sensitivity } \\
{[95 \% \mathrm{Cl}]}\end{array}$ & $\begin{array}{l}\text { Specificity } \\
{[95 \% \mathrm{Cl}]}\end{array}$ & $\begin{array}{l}\text { PPV } \\
{[95 \%} \\
\mathrm{Cl}]\end{array}$ & $\begin{array}{l}\text { NPV } \\
{[95 \%} \\
\text { Cl] }\end{array}$ & $\begin{array}{l}\text { Youden } \\
\text { Index J }\end{array}$ & $\begin{array}{l}\text { AUC } \\
{[95 \% \mathrm{Cl}]}\end{array}$ & P-value \\
\hline \multirow[t]{2}{*}{ FGSI } & $\geq 3$ & 81.82 & 90.48 & 69.2 & 95 & 0.7229 & 0.859 & $<0.0001$ \\
\hline & & $\begin{array}{l}{[48.2-} \\
97.7]\end{array}$ & $\begin{array}{l}{[77.4-} \\
97.3]\end{array}$ & $\begin{array}{l}{[46.0-} \\
85.6]\end{array}$ & $\begin{array}{l}{[84.4-} \\
98.5]\end{array}$ & & $\begin{array}{l}{[0.736-} \\
0.939]\end{array}$ & \\
\hline \multirow[t]{2}{*}{ UFGSI } & $\geq 4$ & 54.55 & 90.48 & 60 & 88.4 & 0.4502 & 0.785 & 0.0014 \\
\hline & & $\begin{array}{l}{[23.4-} \\
83.3]\end{array}$ & $\begin{array}{l}{[77.4-} \\
97.3]\end{array}$ & $\begin{array}{l}{[33.8-} \\
81.5]\end{array}$ & $\begin{array}{l}{[79.8-} \\
93.6]\end{array}$ & & $\begin{array}{l}{[0.650-} \\
0.886]\end{array}$ & \\
\hline \multirow[t]{2}{*}{ SOFA } & $\geq 4$ & 81.82 & 88.1 & 64.3 & 94.9 & 0.6991 & 0.931 & $<0.0001$ \\
\hline & & $\begin{array}{l}{[48.2-} \\
97.7]\end{array}$ & $\begin{array}{l}{[74.4-} \\
96.0]\end{array}$ & $\begin{array}{l}{[43.0-} \\
81.1]\end{array}$ & $\begin{array}{l}{[84.0-} \\
98.5]\end{array}$ & & $\begin{array}{l}{[0.826-} \\
0.982]\end{array}$ & \\
\hline \multirow[t]{2}{*}{$\mathrm{ACCl}$} & $\geq 8$ & 100 & 88.1 & 68.7 & 100 & 0.8810 & 0.969 & $<0.0001$ \\
\hline & & $\begin{array}{l}{[71.5-} \\
100.0]\end{array}$ & $\begin{array}{l}\text { [74.4- } \\
96.0]\end{array}$ & $\begin{array}{l}{[49.1-} \\
83.4]\end{array}$ & {$[-]$} & & $\begin{array}{l}{[0.880-} \\
0.997]\end{array}$ & \\
\hline
\end{tabular}

All data are presented in Median values [Min-Max] 
FGSI: Fournier Gangrene Severity Index, UFGSI: Uludag Fournier Gangrene Severity Index, SOFA: Sequential Organ Failure System, ACCl: Age-Adjusted Charlson Comorbidity Index

\section{Figures}

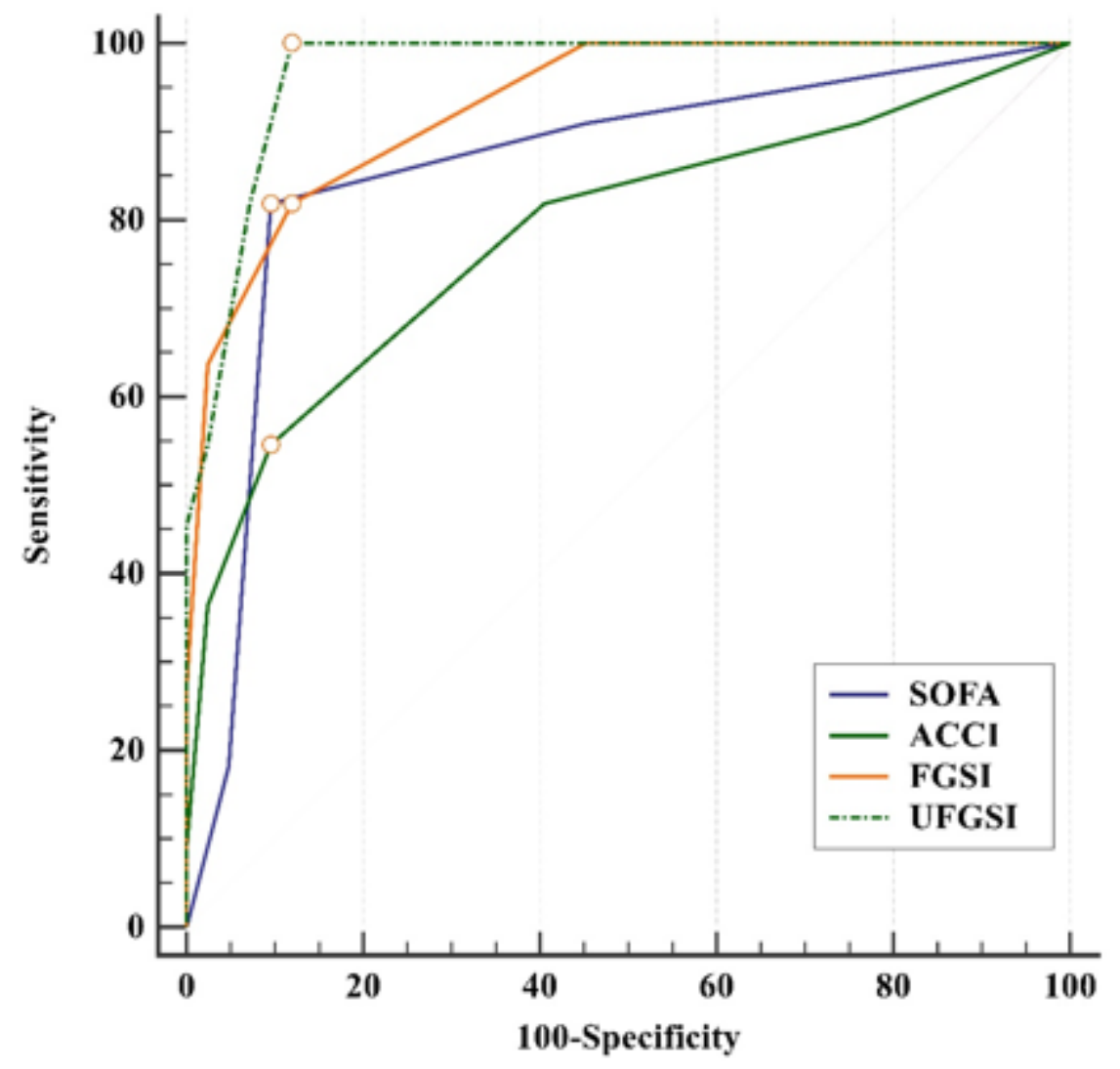

\section{Figure 1}

The combined receiver operating characteristic curves of four scoring systems to predict the mortality of Fournier's gangrene patients. 